\title{
Fidgeting Behavior During Psychotherapy: Hand Movement Structure Contains Information About Depressive Symptoms
}

\author{
Katharina C. H. Reinecke ${ }^{1}$ (D) $\cdot$ Daniela Dvoretska ${ }^{1} \cdot$ Peter Joraschky $^{2} \cdot$ Hedda Lausberg $^{1}$
}

Published online: 7 July 2020

(c) The Author(s) 2020

\begin{abstract}
Fidgeting may be a motor sign reflecting self-regulation processes in patients with social anxiety disorder (SAD) and depressive patients. Since SAD co-occurs with comorbid depression, the question arises whether fidgeting is a disorder-unspecific phenomenon or a specific and therefore diagnostically relevant sign of depression. 33 SAD patients with $(n=12)$ and without $(\mathrm{n}=21)$ depression from the Social Phobia Psychotherapy Research Network project were compared regarding their nonverbal behavior. Four video sequences of a psychotherapy session with each patient were analyzed using a standardized system for the analysis of nonverbal behavior by two independent, certified, blind raters. SAD patients with comorbid depression exhibited significantly more (number/minute) irregular movements, but fewer (number/minute) repetitive movements than SAD patients without depression. Irregular movements, which reflect less structured motor behavior, are associated with comorbid depression in SAD. In contrast, in SAD without depression, more structured repetitive movements were prominent. Thus, irregular movements represent a diagnostically relevant behavior for comorbid depression among SAD patients.
\end{abstract}

Keywords Fidgeting $\cdot$ Irregular movements $\cdot$ Social anxiety disorder $\cdot$ Depression $\cdot$ Repetitive movements

\section{Introduction}

In outpatient psychotherapy, adopting a new perspective and seeing oneself from the observer's point of view by employing self-observation (Koffert et al. 2019) can be helpful. During a psychotherapy session, therapists take on the observer's point of view by focusing their attention on their patients. In doing so, therapists focus not only on the verbal content but also on their patients' nonverbal behavior. Fidgeting is a prominent observable movement behavior in patients with social anxiety disorder (SAD) and persons with subclinical social anxiety and arousal. Fidgeting is associated with SAD, as irregular hand movements, or continuous small-amplitude movements with the hands, were found to decrease over time in ten SAD patients (Kreyenbrink et al.

Katharina C. H. Reinecke

k.reinecke@dshs-koeln.de

1 Department of Neurology, Psychosomatic Medicine and Psychiatry, German Sport University Cologne, Am Sportpark Muengersdorf 6, Cologne 50933, Germany

2 Clinic for Psychotherapy and Psychosomatics, Carl Gustav Carus University Dresden, Fiedlerstrasse 19, Dresden 01307, Germany
2017). Fidgeting has also been linked to stress and arousal in healthy persons (Densing et al. 2018). Heerey and Kring (2007) identified a socially anxious group and a non-socially anxious group in a student sample. During an interview situation with a stranger, individuals in the socially anxious group displayed increased fidgeting.

Patients with depression likewise display fidgeting behaviors (Freedman and Hoffman, 1967; Scherer et al. 2014), which also decrease over the course of successful treatment (Ulrich and Harms 1985; Lausberg and Kryger 2011). Since the comorbidity for depression and anxiety is high (47\%; Stein et al. 2017), it remains unclear whether fidgeting is a specific phenomenon of SAD or depression.

One explanation for the diversity of findings is the usage of different methodologies and a notable lack of uniformity in operationalizations. The American Psychiatric Association lists "psychomotor agitation" as a motor criterion for the diagnosis of major depression in DSM-V (American Psychiatric Association 2013). Fidgeting has been defined in a diversity of ways, such as psychomotor agitation of the hands (Heerey and Kring 2007); "average acceleration from both feet" (Belak et al. 2017; p.3); "the total number of movements observed" (Farley et al. 2013; p.3), further distinguished into "head movements, appendage movements 
or body movements" (Farley et al. 2013; p.3) or "stammers, trembling, or fidgets" (Dow 1985). These diverse definitions and resulting operationalizations are one reason why descriptions of movement behavior in clinical settings are often vague. In turn, these different operationalizations may contribute to the contradictory results found in previous research. Moreover, fidgeting also occurs in other mental diseases and syndromes, such as eating disorders (Belak et al. 2017) or alexithymia (Lausberg et al. 2016), as well as in dysfunctional neurological states, such as patients who have experienced a concussion (Helmich and Lausberg 2019). Fidgeting, therefore, might instead be an unspecific motor phenomenon associated with mental disorders in general.

Among the fidgeting movements, those of the hands are of specific interest for diagnostic purposes during clinical interviews. Based on cinematographic and microanalysis studies, researchers differentiate between two types of hand fidgeting movements: repetitive movements and irregular movements (Lausberg 2013; Lausberg and Sloetjes 2016). Repetitive movements encompass a specific trajectory pattern. This trajectory pattern comprises three phases: a transport phase, in which the hand moves to the location where it engages in the action; a complex phase, in which the hand moves repeatedly forth and back on the same path, e.g., stroking the back of the hand; and a retraction phase, in which the hand moves back to rest position. In contrast, irregular movements have no phase structure. The hand movement starts and ends wherever the hand happens to be. These are typically small movements, and their trajectory lacks any clear spatial direction, e.g., fiddling with one's fingers. While repetitive movements are more structured and based on a motor plan, irregular movements are unstructured and seem to happen by themselves, without any motor plan.

Irregular movements in healthy persons are associated with self-regulatory functions while speaking (Helmich et al. 2014) and with cognitive stress (Heubach 2016). The duration (seconds/unit) of irregular movements is associated with post-concussive symptoms (Helmich and Lausberg 2019). A different correlational pattern has been found for repetitive movements: Persons display them when externalizing mental concepts (Helmich et al. 2014). The duration of repetitive right-hand movements is associated with less cognitive stress during the Stroop task (Heubach 2016). Alexithymia is associated with less repetitive movements (Lausberg et al. 2016), while Korean and German dancers transport emotional expression in ballet through repetitive movements (Kim and Lausberg 2019). Fidgeting with a repetitive structure (e.g., scratching) is used to cope with stressful situations (Sousa-Poza and Rohrberg 1977; Grunwald et al. 2014). Furthermore, repetitive fidgeting has been theoretically related to self-regulation (Freedman et al. 1972;
Densing et al. 2018). In mentally healthy students, fidgeting movements serve as a strategy for the successful retention of lecture material (Farley et al. 2013). Mothers' fidgeting through contact with their infants is known to affect infants' stress levels (Waters et al. 2017). Fidgeting as the repetitive circular or back-and-forth rubbing of the back of one hand on the other evokes neural mechanisms related to coping in the brain (Kikuchi and Noriuchi 2019). Thus, repetitive movements appear to indicate highly structured self-regulation processes, while irregular movements do not.

In the present study, a sample of outpatient psychotherapy patients' movement behavior during the first psychotherapy session was analyzed with the behavior analysis system NEUROGES®-ELAN. The patients were diagnosed with SAD and with or without comorbid depression. The cited studies that used the NEUROGES $®$ system to investigate movement behavior suggest a clear conceptualization involving a differentiation between repetitive and irregular movement structure. Therefore, we hypothesized that a difference in the movement structure, namely increased irregular and decreased repetitive hand movements, would be indicative of specific comorbid depressive symptoms. In movement science, Kendon (2004) proposed the definition of movement as "the articulators are moved away from some position of rest or relaxation [...] and then, eventually, they are moved back again [...]" (Kendon 2004; p. 11). Some of the cited studies did not differentiate among more fine-grained movement structures but rather observed movement activation according to this definition. As NEUROGES ${ }^{\circledR}$ enables the user to analyze movement activation, we decided to include an analysis of activation into this study, as well. We hypothesized no differences in hand activation between the two groups.

\section{Methods}

\section{Sample}

The sample consisted of 33 patients with the primary diagnosis SAD (Social Anxiety Disorder; DSM-V, American Psychiatric Association 2013; ICD-10 code: F40.1). Diagnosis was based on the Structured Clinical Interview for Axis I Disorders (SCID-I; First et al. 2015) and was conducted by trained, independent clinicians before the therapy began. Exclusion criteria for the participation in the present study were: psychotic episode, substance use disorder, suicidal or self-harming behavior, personality disorder, organic disorder, high bodily impairment, or ongoing psychotherapeutic treatment. Inclusion criteria were: SAD as the primary diagnosis, age between 18 and 70 years, and no current treatment. SAD (Stein et al. 2017) and depression (Salk et al. 2017) are associated with the female gender. SAD occurs 
with high comorbidities (47\% lifetime comorbidity for a mood disorder; Stein et al. 2017). The inclusion of comorbid disorders and the proportion of female patients $(75 \%$ in this sample) makes this sample comparable to other samples. Participation in the study involved video-recording the patients' therapy sessions (see Video data). The participants gave their written consent to participate in the study, including the use of their video material in research.

Two patient groups were created to investigate the correlations between comorbid depression in SAD patients and motor behavior. A score of 13 on the Beck Depression Inventory-II (BDI-II) pre-measurement screening was used as a cut-off score to differentiate patients with SAD without significant comorbid depressive symptoms from SAD patients with comorbid depressive symptoms (Beck et al. 1996). This cut-off score is in line with the criteria set by the developers of the questionnaire and the German national S3 guidelines for the diagnosis and treatment of unipolar depression (Schneider et al. 2017). As the BDIII measures level of depression $(<13$ : no/minimal depression; $\geq 13$ : depression, which can be further categorized into mild/moderate/severe depression), the scores give insight into the patients' level of impairment in terms of depressive symptoms.

The first group consisted of ten female patients and two male patients with a patient BDI-II-score of $\geq 13$, indicating depression (Beck et al. 1996) at pre-measurement. The second group consisted of fifteen female patients and six male patients with a patient BDI-II-score $<13$. The patients' mean ages ranged between 31 and 39 years (group with depression: $M=39.40$ years, $\mathrm{SD}=14.13$ years; group without depression: $M=31.28$ years, $\mathrm{SD}=9.15$ years). The therapists' ages ranged between 25 and 60 years $(M=37.18$ years, $\mathrm{SD}=11.22$ years).

The patients' level of impairment in terms of SAD symptoms was measured with the LSAS (Liebowitz Social Anxiety Scale; Von Glieschinsky et al. 2018). $\chi^{2}$-Tests for gender, age, and $\mathrm{SAD}$ yielded no significant group differences.

\section{Video Data}

The video data comprise video recordings of naturalistic psychodynamic psychotherapy sessions with the 33 patients from the Social Phobia Psychotherapy Research Network project, SOPHO-NET (Leichsenring et al. 2013). They were recorded in 2008-2009 at the study center of the Department for Psychotherapy and Psychosomatics at the University Hospital of Dresden, Germany. All participants gave their written consent for the use of the video material in research (see Sample).

We chose to analyze video data from the first probationary session for each of the 33 patients. In ambulatory psychotherapy, the probationary therapy session represents the initial meeting. The patient talks about his or her symptoms, while the therapist conducts the diagnosis. Furthermore, as the probationary session represents the first encounter between patient and therapist, neither conversation partner's motor behavior is influenced by prior sessions. Thus, the first session provides insight into the patients' motor behavior before treatment. We chose the first five minutes of each session for the microanalysis, as an excerpt of just a few minutes provides reliable insight into movement behavior (Ambady and Rosenthal 1992). The analyzed video excerpts for the 33 patients were then split into four sequences, resulting in 132 randomized video sequences.

\section{Measurements of Fidgeting}

The NEUROGES $®$ system (Lausberg and Sloetjes 2016; Lausberg 2019) is used as the reference system in the present paper due to its high degree of differentiation and operationalization. In NEUROGES, fidgeting hand movements have either an irregular (e.g., small uneven movements by one finger on the other hand) or repetitive (e.g., itching, scratching) structure (Lausberg 2019; see Introduction). In this paper, we focus on fidgeting distinguished into either irregular or repetitive movements.

The NEUROGES $®$ system is an objective, reliable, and valid interdisciplinary research tool (Lausberg and Sloetjes 2016; Lausberg 2019) for the analysis of motor behavior. NEUROGES ${ }^{\circledR}$ consists of an assessment algorithm grouped into three modules (for detailed information, see Lausberg 2019) that has proven suitable for the analysis of motor behavior in therapeutic interactions (Lausberg and Kryger 2011; Kreyenbrink et al. 2017). In contrast to other movement coding systems (e.g., MEA, Paulick et al. 2017), this system provides a fine-grained descriptive analysis of different kinds of fidgeting, i.e., repetitive versus irregular movements. NEUROGES ${ }^{\circledR}$-ELAN enables the user to analyze the frequency of a given behavioral unit, such as a repetitive or an irregular unit (measured in number of units per minute).

In this study, we used NEUROGES ${ }^{\circledR}$ Module I (analysis of hand movements using the NEUROGES ${ }^{\circledR}$ Activation and Structure categories), as it fits our research question. NEUROGES $®$ Structure values (irregular, repetitive, phasic, shift, and aborted) register the structure of a movement. In contrast to irregular movements, phasic and repetitive movements are characterized by a phase structure, beginning with a preparation phase, followed by a complex phase with either a phasic or repetitive movement trajectory (Lausberg 2019), and typically terminated by a retraction phase.

\section{Coding Procedure and Inter-Rater Reliability}

NEUROGES-certified raters analyzed the video sequences using the following standard procedure (Lausberg 2013): The 
first rater coded $100 \%$ of the video sequences, and the second rater coded $25 \%$ of all video sequences. Both raters coded the data independently, blind to the patient groups and diagnoses, as well as without an audio track to prevent coding biases based on the recorded sounds and conversations.

Coding was conducted on the 132 previously identified video sequences (see Video data). Furthermore, the order of all 132 video sequences was pseudo-randomized for each rater. We repeated this procedure for each NEUROGES ${ }^{\circledR}$ category (Activation, and Structure).

To calculate the inter-rater reliability for the Activation category, we used the merge-overlap ratio (Lausberg 2013). To calculate the inter-rater reliability for the Structure category, we used a modified Cohen's Kappa (Holle and Rein 2015) considering agreement on the values as well as agreement on the segmentation of the behavior (Holle and Rein 2015). Merge-overlap-ratios for the Activation category ranged between 0.83 and 0.87. Cohen's Kappa for the Structure category ranged between 0.57 and 0.80 . Using Landis \& Koch as a point of reference, the inter-rater reliability in this study is "substantial" to "almost perfect" for the Activation category and "moderate" to "almost perfect" for the Structure category (Landis and Koch 1977).

\section{Statistical Analyses}

We conducted an ANOVA for the Activation category. We chose "hand" as a within-subjects factor because the left and right hands are analyzed separately in NEUROGES $®$ Module I. We calculated a MANOVA for the frequency (frequency describes the mean number of value units per minute; see Lausberg 2019) of hand movements for the Structure category due to the interdependent nature of the dependent variable (e.g., frequency of the Structure values irregular/repetitive/ phasic/shift), the between-subjects factor "group" (comorbid depressive patients with BDI-II $\geq 13$ at pre-measurement vs. vs. non-depressive patients with BDI-II $<13$ at pre-measurement) and the within-subjects factor "hand" (e.g., irregular left-handed movement, irregular right-handed movement). The calculation included a Bonferroni adjustment to correct for multiple testing. For each NEUROGES ${ }^{\circledR}$ category, the frequency distribution of the values is reported. Only values that were displayed by at least ten persons in each group were included in the analysis. We conducted data analyses with SPSS Version 25. Taking a bottom-up approach, we first analyzed movement activation and then movement structure and therefore report the results in this order.

\section{Results}

\section{Activation}

\section{Frequency Distributions of the Hand Movements}

All 33 patients displayed movement units.

\section{Frequency (number/minute)}

ANOVA for the frequency of movement units revealed no significant group differences.

\section{Structure}

\section{Frequency Distributions of the Structure Values Phasic, Repetitive, Irregular, Shift, and Aborted}

The Structure values phasic, repetitive, irregular, and shift were displayed by more than ten persons in each group and therefore included in the analysis. Aborted units were displayed by fewer than ten persons in each group and were therefore excluded from the analysis, which is a common finding in studies using NEUROGES® (see Lausberg 2019).

\section{Frequency (number/minute)}

The multivariate between-subjects tests showed a non-significant trend of group on the frequency (number/minute) of the four Structure values $(F(4 / 28)=2.68 ; p=0.052$; partial $\eta^{2}=0.28$ ). The univariate tests showed a significant betweensubject effect of BDI-II group on the frequency (number/ minute) of irregular units $(F(1 / 31)=5.72 ; p=0.023$; partial $\left.\eta^{2}=0.16\right)$. Comorbid depressive patients exhibited significantly more irregular movements $(M=2.64, S E=0.38$ [95\%-CI $1.873 ; 3.410])$ than patients without comorbid depression $(M=1.51, S E=0.285$ [95\%-CI 0.931; 2.093]). The univariate between-subjects tests revealed a significant effect of BDI-II group on the frequency (number/minute) of repetitive units $\left(F(1 / 31)=6.63 ; p=0.015 ;\right.$ partial $\left.\eta^{2}=0.18\right)$. Comorbid depressive patients exhibited significantly fewer repetitive movements ( $M=0.85, S E=0.26$ [95\%-CI $0.315 ; 1.385])$ than patients without comorbid depression $(M=1.70, S E=0.20$ [95\%-CI 1.292; 2.101], see Table 1).

\section{Discussion}

In the present study, comorbid depressive SAD patients exhibited more irregular movements and fewer repetitive movements than SAD patients without comorbid depression. 
Table 1 Mean frequency (number/minute) and standard errors of patients' overall movements (Activation) and of patients' movement structures, $* F(1 / 31)=5.72 ; p=.023 ;$ partial $\eta^{2}=0.16 p<.05 ; * * F$ $(1 / 31)=6.63 ; p<.05 ;$ partial $\eta^{2}=0.18$

\begin{tabular}{|c|c|c|c|c|}
\hline & \multicolumn{2}{|c|}{$\begin{array}{l}\text { SAD patients with } \\
\text { depression }(N=12)\end{array}$} & \multicolumn{2}{|c|}{$\begin{array}{l}\text { SAD patients } \\
\text { without depression } \\
(N=21)\end{array}$} \\
\hline & $M$ & $S E$ & $M$ & $S E$ \\
\hline Activation & 5.46 & 0.61 & 3.90 & 0.68 \\
\hline Irregular $(*)$ & 2.64 & 0.38 & 1.51 & 0.29 \\
\hline Phasic & 2.66 & 0.42 & 2.95 & 0.32 \\
\hline Repetitive $(* *)$ & 0.85 & 0.26 & 1.70 & 0.12 \\
\hline Shift & 0.78 & 0.18 & 0.56 & 0.14 \\
\hline
\end{tabular}

In line with our hypotheses, the results revealed significant group differences for the specific values irregular and repetitive.

Moreover, SAD patients with comorbid depressive symptoms did not differ in overall movement activity from SAD patients without comorbid depressive symptoms. This finding provides support to the hypothesis that comorbid depression is not reflected in the total amount of motor activity, as measured by movement units with the NEUROGES ${ }^{\circledR}$ Activation category, but rather in the kind of movements, specifically the movement structure as measured in the NEUROGES ${ }^{\circledR}$ Structure category. In other words, SAD patients with comorbid depression are not hyper- or hypoactive as compared to patients without comorbid depression; however, they exhibit specific differences related to the frequency of certain movement structures.

Thus, comorbid depression in SAD patients cannot be distinguished solely by registering hyper- or hypoactivity. Instead, in line with the hypothesis that comorbid depression is associated with an increase of irregular hand movements, the present study demonstrated that SAD patients with comorbid depressive symptoms displayed more irregular movements than SAD patients without comorbid depressive symptoms. Therefore, irregular hand movements can be considered as a specific phenomenon of comorbid depression. As these movements are related to self-regulatory functions and cognitive stress (see Introduction), they probably reflect a particular depressionrelated emotional state. A further look at the results on the structural level revealed fewer repetitive movements in SAD patients with comorbid depression compared to SAD patients without depression. Repetitive movements are related to the externalization of mental concepts, lower cognitive stress, lower alexithymia, emotional expression in dancers, coping with stressful situations, and to self-regulation (see Introduction). Repetitive movements, therefore, indicate highly structured self-regulation processes, which are not common in patients with comorbid depression. Consequently, the present results concerning irregular and repetitive movements are consequently in line with the existing research.

However, some studies have linked irregular movements to social anxiety, not to depression (see Introduction). An explanation for these observations could be that previous studies did not consider comorbidities, and therefore unintentionally included comorbid depressive persons in their SAD samples. Kreyenbrink et al. (2017) did not differentiate SAD patients according to their comorbidities. Likewise, Heerey and Kring (2007) did not screen their participants for depression. As the probability of a comorbidity in SAD patients is $47 \%$ (Stein et al. 2017), some of the participating students could likewise have been persons with depression.

Movements with a repetitive structure are characterized by a higher degree of organization as compared to movements with an irregular structure. This higher degree of structure implies that repetitive movements are more effective at achieving an internal (or external) goal, such as copying and self-regulation, compared to irregular movements. The present findings thus enrich the results of earlier studies (Freedman and Hoffman 1967; Ulrich and Harms 1985) reporting continuous movements in patients with depression, as the present findings differentiate these movements into irregular and repetitive movements, which correlate with different depression-related and depression-unrelated mental states.

This study is the first microanalysis of fidgeting hand movements using an operationalization differentiating between irregular and repetitive movement structures. Due to the use of naturalistic data, groups in this study were not equal in size, and the resulting representative sample is small. Therefore, the results should be interpreted as preliminary. Future studies should test whether irregular movements are, in addition to depressive symptoms, associated with other disease-related features in addition to depressive symptoms, such as the outcome of the psychotherapeutic intervention.

The present results from naturalistic psychotherapy settings confirm the importance of analyzing the structure of movement behavior. Thus, the criterion "psychomotor agitation", which is the motor criterion of the diagnosis major depression in the DSM-V, should be further specified. By taking an observer's point of view, the psychotherapist can differentiate the patient's movement structures during an ongoing psychotherapy session. Notably, more irregular movements and a lack of repetitive movements are present in SAD patients with comorbid depression during the first psychotherapy session. As a consequence, practitioners experienced with motor behavior observation should be able to identify correlates of depressive comorbidity in their patients during the first diagnostic interview. 
Acknowledgements The authors would like to thank Keri Hartman for English proofreading.

Author Contributions PJ has made substantial contributions to the acquisition of the data. DD and KR have substantially analyzed the data. KR drafted the manuscript. KR and HL have substantively revised the manuscript.

Funding Open access funding provided by Projekt DEAL. This research did not receive any specific grants from funding agencies in the public, commercial, or not-for-profit sectors.

Data Availability The datasets used and/or analyzed during the current study are available from the corresponding author upon reasonable request.

\section{Compliance with Ethical Standards}

\section{Conflicts of interest None.}

Ethics Approval and Ethical Standards The ethical board of the GSU Cologne approved this study. All procedures performed involving human participants were following the ethical standards of the institutional research committee and with the 1964 Declaration of Helsinki and its later amendments or comparable ethical standards.

Informed Consent Informed consent was obtained from all participants included in the study.

Open Access This article is licensed under a Creative Commons Attribution 4.0 International License, which permits use, sharing, adaptation, distribution and reproduction in any medium or format, as long as you give appropriate credit to the original author(s) and the source, provide a link to the Creative Commons licence, and indicate if changes were made. The images or other third party material in this article are included in the article's Creative Commons licence, unless indicated otherwise in a credit line to the material. If material is not included in the article's Creative Commons licence and your intended use is not permitted by statutory regulation or exceeds the permitted use, you will need to obtain permission directly from the copyright holder. To view a copy of this licence, visit http://creativecommons.org/licenses/by/4.0/.

\section{References}

Ambady, N., \& Rosenthal, R. (1992). Thin slices of expressive behavior as predictors of interpersonal consequences: A meta-analysis. Psychological Bulletin, 111(2), 256.

American Psychiatric Association. (2013). Diagnostic and Statistical Manual of Mental Disorders (DSM-5®). American Psychiatric Pub.

Beck, A. T., Steer, R. A., \& Brown, G. K. (1996). Beck depression inventory-II. San Antonio, 78(2), 490-498.

Belak, L., Gianini, L., Klein, D. A., Sazonov, E., Keegan, K., Neustadt, E., et al. (2017). Measurement of fidgeting in CC nervosa using a novel shoe-based monitor. Eating Behaviors, 24, 45-48.

Densing, K., Konstantinidis, H., \& Seiler, M. (2018). Effect of stress level on different forms of self-touch in pre-and postadolescent girls. Journal of Motor Behavior, 50(5), 475-485.

Dow, M. G. (1985). Peer validation and idiographic analysis of social skill deficits. Behavior Therapy, 16(1), 76-86.
Farley, J., Risko, E., \& Kingstone, A. (2013). Everyday attention and lecture retention: The effects of time, fidgeting, and mind wandering. Frontiers in Psychology, 4, 619.

Freedman, N., \& Hoffman, S. P. (1967). Kinetic behavior in altered clinical states: Approach to objective analysis of motor behavior during clinical interviews. Perceptual and Motor Skills, 24(2), 527-539.

Freedman, N., O’Hanlon, J., Oltman, P., \& Witkin, H. A. (1972). The imprint of psychological differentiation on kinetic behavior in varying communicative contexts. Journal of Abnormal Psychology, 79(3), 239.

First M.B., Williams J.B.W., Karg R.S, Spitzer R.L. (2015). Structured clinical interview for DSM-5- research version (SCID-5 for DSM-5, research version; SCID-5-RV, version 1.0.0). Arlington: American Psychiatric Association.

Grunwald, M., Weiss, T., Mueller, S., \& Rall, L. (2014). EEG changes caused by spontaneous facial self-touch may represent emotion regulating processes and working memory maintenance. Brain Research, 1557, 111-126.

Heerey, E. A., \& Kring, A. M. (2007). Interpersonal consequences of social nxiety. Journal of Abnormal Psychology, 116(1), 125.

Helmich, I., Skomroch, H., \& Lausberg, H. (2014). Neuropsychological functions of hand movements and gestures change in the presence or absence of speech. Journal of Cognitive Psychology, 26(7), 740-753.

Helmich, I., \& Lausberg, H. (2019). Nonverbal hand movement durations indicate post-concussion symptoms of athletes. Journal of Neurotrauma, 36(20), 2913-2921.

Heubach T. (2016). Auswirkungen von kognitivem Stress auf das Handbewegungsverhalten- Können wir durch Handbewegungen während kognitiver Tests unsere Leistung steigern? [Effects of cognitive stress on hand movement behavior - Can we improve our performance during cognitive tests using hand movements?] German Sport University Cologne.

Holle, H., \& Rein, R. (2015). EasyDIAg: A tool for easy determination of interrater agreement. Behavior Research Methods, 47(3), $837-847$.

Kendon A. (2004). Gesture: Visible action as utterance. Cambridge University Press.

Kikuchi Y., Noriuchi M. (2019). Power of Self-Touch: Its Neural Mechanism as a Coping Strategy. In Emotional Engineering, Vol. 7 (pp. 33-47) Cham: Springer.

Kim Z.H., Lausberg, H. (2019). Processing Emotional Expression in the Dance of a Foreign Culture: Gestural Responses of Germans and Koreans to Ballet and Korean Dance. Journal of Movement Arts Literacy, 4(1).

Kreyenbrink I, Joraschky P, Konstantinidis I, Neumann N, Lausberg H. (2017). Nonverbales Verhalten von Patienten mit sozialen Phobien und ihren Therapeuten in psychodynamischen Psychotherapien (Teilprojekt SOPHO-NET). Zeitschrift für Psychosomatische Medizin und Psychotherapie, 297-313.

Koffert, T., Luutonen, S., Niemi, P. M., Tiuraniemi, J., Nordström, E., Keinänen, M., et al. (2019). Patient-made videos as a tool of selfobservation enhancing self-reflection in psychotherapy. Journal of Contemporary Psychotherapy, 49(3), 187-195.

Landis J.R., Koch G.G. (1977). An application of hierarchical kappatype statistics in the assessment of majority agreement among multiple observers. Biometrics, 363-374.

Lausberg, H., \& Kryger, M. (2011). Gestisches Verhalten als Indikator therapeutischer Prozesse in der verbalen Psychotherapie: Zur Funktion der Selbstberührungen und zur Repräsentation von Objektbeziehungen in gestischen Darstellungen. PsychotherapieWissenschaft, 1(1), 41-55.

Lausberg H. (2013). Understanding Body Movement: A Guide to Empirical Research on Nonverbal Behaviour-With an Introduction 
to the NEUROGES®Coding System. Peter Lang International Academic Publishers.

Lausberg, H., Helmich, I., Sassenberg, U., \& Petermann, K. (2016). Gender-specific implicit emotional processing in alexithymic individuals. Journal of Psychosomatic Research, 85, 70.

Lausberg, H., \& Sloetjes, H. (2016). The revised NEUROGES-ELAN system: An objective and reliable interdisciplinary analysis tool for nonverbal behavior and gesture. Behavior Research Methods, 48(3), 973-993.

Lausberg H. (2019). The NEUROGES® Analysis System for Nonverbal Behavior and Gesture: The complete research coding manual including an interactive video learning tool and coding template. Peter Lang $\mathrm{GmbH}$.

Leichsenring, F., Salzer, S., Beutel, M. E., Herpertz, S., Hiller, W., Hoyer, J., et al. (2013). Psychodynamic therapy and cognitivebehavioral therapy in social anxiety disorder: A multicenter randomized controlled trial. American Journal of Psychiatry, 170(7), 759-767.

Paulick, J., Deisenhofer, A. K., Ramseyer, F., Tschacher, W., Boyle, K., Rubel, J., et al. (2017). Nonverbal synchrony: A new approach to better understand psychotherapeutic processes and drop-out. Journal of Psychotherapy Integration, 28(3), 367.

Salk, R. H., Hyde, J. S., \& Abramson, L. Y. (2017). Gender differences in depression in representative national samples: Meta-analyses of diagnoses and symptoms. Psychological Bulletin, 143(8), 783.

Scherer, S., Stratou, G., Lucas, G., Mahmoud, M., Boberg, J., Gratch, J., et al. (2014). Automatic audiovisual behavior descriptors for psychological disorder analysis. Image and Vision Computing, 32(10), 648-658.
Schneider F., Härter M., Schorr S. (Eds.). (2017). S3-Leitlinie/Nationale VersorgungsLeitlinie Unipolare Depression. Verlag : Springer.

Stein, D. J., Lim, C. C., Roest, A. M., De Jonge, P., Aguilar-Gaxiola, S., Al-Hamzawi, A., et al. (2017). The cross-national epidemiology of social anxiety disorder: Data from the World Mental Health Survey Initiative. BMC Medicine, 15(1), 143.

Sousa-Poza, J. F., \& Rohrberg, R. (1977). Body movement in relation to type of information (person-and nonperson-oriented) and cognitive style (field dependence). Human Communication Research, $4(1), 19-29$.

Ulrich, G., \& Harms, K. (1985). A video analysis of the non-verbal behaviour of depressed patients before and after treatment. Journal of Affective Disorders, 9(1), 63-67.

Von Glischinski, M., Willutzki, U., Stangier, U., Hiller, W., Hoyer, J., Leibing, E., et al. (2018). Liebowitz Social Anxiety Scale (LSAS): Optimal cut points for remission and response in a German sample. Clinical Psychology \& Psychotherapy, 25(3), 465-473.

Waters, S. F., West, T. V., Karnilowicz, H. R., \& Mendes, W. B. (2017). Affect contagion between mothers and infants: Examining valence and touch. Journal of Experimental Psychology: General, 146(7), 1043.

Publisher's Note Springer Nature remains neutral with regard to jurisdictional claims in published maps and institutional affiliations. 